\title{
Stochastic particle approximations for the Ricci flow on surfaces and the Yamabe flow
}

\author{
Robert Philipowski ${ }^{* \dagger}$
}

\begin{abstract}
We present stochastic particle approximations for the normalized Ricci flow on surfaces and for the non-normalized Yamabe flow on manifolds of arbitrary dimension.
\end{abstract}

Keywords: Ricci flow on surfaces, Yamabe flow, stochastic interacting particle system. AMS subject classification: $53 \mathrm{C} 44,82 \mathrm{C} 22$.

\section{Ricci and Yamabe flow}

Thanks to Perelman's seminal work on the geometrization and hence the Poincaré conjecture $[19,20,21]$ the Ricci flow has attracted worldwide attention and led to many new developments (see e.g. [17]). The importance of this subject has been underlined by the award of the Fields medal to Perelman. There is now a strong interest in understanding the microscopic structure of the Ricci flow.

The evolution of a Riemannian metric $g=g_{t}$ on a connected $d$-dimensional closed manifold $M$ under the (normalized) Ricci flow is described by the partial differential equation

$$
\frac{\partial g}{\partial t}=\frac{2}{d} \bar{R} g-2 \text { Ric . }
$$

Here Ric is the Ricci curvature and $\bar{R}$ the average scalar curvature of $M$, i.e. $\bar{R}:=\frac{1}{\operatorname{vol}(M)} \int_{M} R$, where $R$ is the scalar curvature (all quantities taken with respect to $g_{t}$ ).

In dimension $d=2$ we have $\operatorname{Ric}=\frac{1}{2} R g$, so that in this case (1) is equivalent to the Yamabe flow

$$
\frac{\partial g}{\partial t}=(\bar{R}-R) g
$$

While in dimension $d \geq 3$ the Ricci flow does not usually admit global solutions (singularities can develop, see e.g. [17]), Hamilton [12] and Chow [5] (see also Theorem 5.1 in [6]) proved the following theorem concerning the 2-dimensional case:

Proposition 1. Let $g_{0}$ be any Riemannian metric on $M$.

1. The Ricci flow equation has a unique solution $\left(g_{t}\right)_{t \geq 0}$ with initial data $g_{0}$.

2. As $t \rightarrow \infty, g_{t}$ converges in any $C^{k}$-norm to a smooth metric $g_{\infty}$ of constant curvature.

For the Yamabe flow in dimension $d \geq 3$ Ye [26] proved global existence and uniqueness for arbitrary initial metrics. Convergence theorems under various assumptions were proved by Ye [26], Schwetlick and Struwe [23] and Brendle [2, 3].

\footnotetext{
*Institut für Angewandte Mathematik, Universität Bonn, Endenicher Allee 60, D-53115 Bonn, Germany.

E-mail address: philipowski@iam.uni-bonn.de

${ }^{\dagger}$ This work was supported by a research grant of the Deutsche Forschungsgemeinschaft.
} 


\section{Stochastic particle approximation for the Ricci flow on sur- faces}

Since by (2) the conformal class of $g$ is conserved under the two-dimensional Ricci flow it is possible to represent $g_{t}$ in the form

$$
g_{t}=u(t, \cdot) \tilde{g} \quad \forall t \geq 0,
$$

where $\tilde{g}$ is a fixed reference metric on $M$ and $u$ a smooth function on $\mathbb{R}_{+} \times M$ (one can for instance take $\tilde{g}=g_{0}$, so that in this case $u_{0}:=u(0, \cdot)=1$ ). This approach has the advantage that now the unknown is no longer a family of metrics, but just a function. In order to derive the partial differential equation satisfied by $u$ we need the following lemma (for a proof see e.g. [6], Lemma 5.3).

Lemma 1. Let $g=u \tilde{g}$. Then there is the following relation between the scalar curvatures with respect to $g$ and $\tilde{g}$ :

$$
R_{g}=\frac{1}{u}\left(-\Delta_{\tilde{g}}(\log u)+R_{\tilde{g}}\right) .
$$

Since moreover by the Gauss-Bonnet theorem $\bar{R}_{g}=4 \pi \chi(M) / \operatorname{vol}_{g}(M)$, it follows that

$$
\frac{\partial u}{\partial t}=\Delta_{\tilde{g}}(\log u)-R_{\tilde{g}}+\frac{4 \pi \chi(M)}{\operatorname{vol}_{g}(M)} u .
$$

Remark 1. As a consequence of the convergence of $g_{t}$ to $g_{\infty}$ (Proposition 1) and the compactness of $M, u$ is uniformly smooth, i.e. $u$ and all its derivatives are uniformly bounded in $t \in \mathbb{R}_{+}$and $x \in M$. Moreover, since $u(t, x)>0$ for all $t \in \mathbb{R}_{+}$and all $x \in M$ (because each $g_{t}$ is a Riemannian metric), Proposition 1 implies that $u_{\text {min }}:=\inf \{u(t, x) \mid t \geq 0, x \in M\}$ is strictly positive.

For the stochastic particle approximation it is essential that the metric $\tilde{g}$ has non-positive curvature.

Assumption 1. $R_{\tilde{g}} \leq 0$ everywhere on $M$.

For simplicity we assume:

Assumption 2. $\operatorname{vol}_{g}(M)=\operatorname{vol}_{\tilde{g}}(M)=1\left(\operatorname{vol}_{g}(M)\right.$ is conserved under the Ricci flow, see e.g. [12], Section 2).

From now on all geometric and analytic quantities on $M$ are taken with respect to the reference metric $\tilde{g}$.

The particle system lives on a set $S_{k}$ of $k$ points of $M$ which is supposed to be approximately uniform in $M$ in the sense that the discrete measure $\mu_{k}:=\frac{1}{k} \sum_{x \in S_{k}} \delta_{x}$ converges weakly to the Riemannian volume measure on $M$ as $k \rightarrow \infty$. By Theorem 11.3.3 in [8] this is equivalent to the property that

$$
D_{k}:=\sup _{f \in B L_{1}}\left|\frac{1}{k} \sum_{x \in S_{k}} f(x)-\int_{M} f(y) d y\right| \rightarrow 0 \quad \text { as } k \rightarrow \infty,
$$

where $B L_{1}$ is the set of all Lipschitz continuous functions on $M$ with $\|f\|_{B L}:=|f|_{\text {lip }}+\|f\|_{\infty} \leq 1$.

Remark 2. One possible choice is $S_{k}:=\left\{\xi_{1}, \ldots, \xi_{k}\right\}$ with independent and uniformly (with respect to the Riemannian volume measure) distributed $M$-valued random variables $\xi_{1}, \ldots, \xi_{k}$. In this case the weak convergence of $\mu_{k}$ to the Riemannian volume measure and hence (4) holds almost surely (Theorem 11.4.1 in [8]). 
The particle system consists of $N$ particles moving as follows in discrete time steps of length $\tau>0$ on $S_{k}$ : Suppose that at time $n \tau$ (i.e. after $n$ time steps) there are exactly $m \geq 1$ particles at a site $x \in S_{k}$. Then each of these particles, independently of the past and of the behaviour of all other particles, behaves as follows: With probability

$$
\pi(x, y):=\frac{\tau}{k}\left[p_{r}(x, y) \varphi(k m / N) / r-R(y)\right]
$$

it jumps to a point $y \neq x$, otherwise it stays at $x$. Here $r>0$ is a parameter and $p_{r}(x, y)$ is the heat kernel on $M$ at time $r$, i.e. the fundamental solution of the heat equation $\frac{\partial p}{\partial t}=\Delta p$. Moreover

$$
\varphi(u):= \begin{cases}\frac{\log u-\log u_{\min }}{u} & \text { if } u \geq u_{\min } \\ 0 & \text { if } u<u_{\min } .\end{cases}
$$

(Recall that $u_{\text {min }}:=\inf \{u(t, x) \mid t \geq 0, x \in M\}>0$, see Remark 1). Note that $\pi(x, y)$ is always nonnegative because of our assumption $R \leq 0$. In order to ensure that $\sum_{y \neq x} \pi(x, y) \leq 1$ (so that the jump rule (5) is well-defined), we will assume that

$$
\tau \leq\left(\frac{1}{r k e u_{\min }} \max _{x \in S_{k}} \sum_{y \in S_{k} \backslash\{x\}} p_{r}(x, y)-\frac{1}{k} \sum_{y \in S_{k}} R(y)\right)^{-1},
$$

where $e$ is Euler's number (note that $\varphi$ is bounded by $\frac{1}{e u_{\min }}$ ). In order to give a formal definition of the particle system we introduce the following notation:

1. The particle configuration at time $n \tau$ (i.e. after $n$ time steps) is denoted by

$$
X_{n}=\left(X_{n}^{1}, \ldots, X_{n}^{N}\right) \in S_{k}^{N} .
$$

This means that after $n$ time steps the $i$-th particle is located at $X_{n}^{i}$.

2. For $\mathbf{x}=\left(x_{1}, \ldots, x_{N}\right) \in S_{k}^{N}$ and $y \in S_{k}$ we define

$$
\overline{\mathbf{x}}(y):=\frac{1}{N} \sum_{i=1}^{N} 1_{x_{i}}(y) .
$$

In particular $\bar{X}_{n}(x)$ is the total mass at $x$ after $n$ time steps if we define the mass of each particle to be $1 / N$.

Using this notation the stochastic dynamics can be exactly described as follows:

1. The particle system is a time-discrete Markov process $\left(X_{n}\right)_{n \in \mathbb{N}_{0}}$ with state space $S_{k}^{N}$.

2. The transition probabilities are as follows:

(a) Independence of the jumps of different particles:

$$
P\left[X_{n+1}=\mathbf{y} \mid X_{n}=\mathbf{x}\right]=\prod_{i=1}^{N} P\left[X_{n+1}^{i}=y_{i} \mid X_{n}=\mathbf{x}\right]
$$

for $\mathbf{x}=\left(x_{i}\right)_{i=1}^{N}, \mathbf{y}=\left(y_{i}\right)_{i=1}^{N} \in S_{k}^{N}$.

(b) Jump probability of each single particle:

$$
P\left[X_{n+1}^{i}=y \mid X_{n}=\mathbf{x}\right]:= \begin{cases}\frac{\tau}{k}\left[p_{r}\left(x_{i}, y\right) \varphi\left(k \overline{\mathbf{x}}\left(x_{i}\right)\right) / r-R(y)\right] & \text { if } y \neq x_{i} \\ 1-\frac{\tau}{k} \sum_{z \in S_{k} \backslash\left\{x_{i}\right\}}\left[p_{r}\left(x_{i}, z\right) \varphi\left(k \overline{\mathbf{x}}\left(x_{i}\right)\right) / r-R(z)\right] & \text { if } y=x_{i}\end{cases}
$$

for $y \in S_{k}$. 
In order to study the macroscopic behaviour of the particle system we consider the empirical measure defined as

$$
\bar{X}_{\tau, r, k}^{N}(t, x):=\bar{X}_{\lfloor t / \tau\rfloor}(x)+\left(\frac{t}{\tau}-\left\lfloor\frac{t}{\tau}\right\rfloor\right)\left[\bar{X}_{\lfloor t / \tau\rfloor+1}(x)-\bar{X}_{\lfloor t / \tau\rfloor}^{N}(x)\right] .
$$

(Here we interpolate piecewise linearly in time).

Theorem 1. There are constants $A_{1}, A_{2}, A_{3}<\infty$ (depending on $(M, \tilde{g})$ and $u_{0}$ ) such that for all $T \geq 0$, all $N, k \in \mathbb{N}$, all $r \in(0,1]$ and all $\tau>0$ satisfying $(6)$ :

$$
\begin{aligned}
& E\left[\sup _{0 \leq t \leq T} \sup _{f \in B L_{1}}\left|\int_{M} f(y) u(t, y) d y-\sum_{x \in S_{k}} f(x) \bar{X}_{\tau, r, k}^{N}(t, x)\right|\right] \\
\leq & E\left[\frac{1}{k} \sum_{x \in S_{k}}\left|u_{0}(x)-k \bar{X}_{0}(x)\right|\right]+T\left[A_{1} \tau+A_{2} r+A_{3} r^{-5 / 2} D_{k}+\frac{k}{2 \tau \sqrt{N}}\right] .
\end{aligned}
$$

This means that the empirical measure of the particle system converges locally uniformly in time to the measure with density $u$ as $N$ and $k$ tend to $\infty$ and $\tau$ and $r$ tend to 0 , provided that the initial configuration is chosen appropriately and the constraints $r^{-5 / 2} D_{k} \rightarrow 0, \frac{k}{\tau \sqrt{N}} \rightarrow 0$ and (6) are respected.

\section{Remarks concerning related work}

Particle approximations to nonlinear diffusion equations have been studied for a long time (see the books by Kipnis and Landim [15] and Spohn [24] for overviews). However, to the author's knowledge, no particle approximation for the Ricci flow has been proposed yet.

Written in the form (3), the Ricci flow equation on a surface is a logarithmic diffusion equation with source term and hence belongs to the class of filtration or generalized porous medium equations (see the book by Vázquez [25] for the analytical theory of this type of equations). For the porous medium equation particle approximations have been constructed by Ekhaus and Seppäläinen [9], Feng, Iscoe and Seppäläinen [10], Figalli and the author [11], Inoue [13], Jourdain [14], Oelschläger [18] and the author [22]. However, in all these papers the particle system and the limit equation are defined on a flat space $\left(\mathbb{R}^{d}\right.$ or the flat torus $\left.T^{d}:=\mathbb{R}^{d} / \mathbb{Z}^{d}\right)$. It seems that in the present paper for the first time a particle approximation for a nonlinear diffusion equation on a non-flat Riemannian manifold is given.

Another related work is the construction of a combinatorial (i.e. discrete) variant of the Ricci flow by Chow and Luo [7] (see also [16]). In their paper the surface is triangulated, and the metric is given as a function which assigns to each vertex $v_{i}(i=1, \ldots, N)$ of the triangulation a positive number $r_{i}$ called radius. The distance between two adjacent vertices is defined as the sum of the two radii, and the triangles are realized geometrically as Euclidean (or even hyperbolic or spherical) triangles. In this case the curvature is concentrated at the vertices, and at the vertex $v_{i}$ it is given as the angle defect $K_{i}:=2 \pi-a_{i}$, where $a_{i}$ is the sum of all angles adjacent to $v_{i}$. The (normalized) combinatorial Ricci flow is then given by the following system of ordinary differential equations:

$$
\frac{d r_{i}}{d t}=-\left(K_{i}-K_{a v}\right) r_{i}
$$

where $K_{a v}:=1 / N \sum_{i=1}^{N} K_{i}$ is the average curvature. The main theorem in [7] is as follows: Under an approriate condition on the triangulation the solution of (7) exists for all time and converges exponentially fast to a metric of constant curvature. 


\section{Proof of Theorem 1}

A crucial role in the proof is played by the discrete Laplacian, defined by

$$
\Delta_{r, k} f(x)=\frac{1}{r k} \sum_{y \in S_{k}} p_{r}(x, y)[f(y)-f(x)] .
$$

The discrete Laplacian is the link between the Laplace-Beltrami operator appearing in equation (3) and the jump probabilities of the particles.

Lemma 2. There is a constant $C(M)<\infty$ such that for all $f \in \mathcal{C}^{4}(M)$, all $x \in M$, all $r \in(0,1]$ and all $k \in \mathbb{N}$

$$
\left|\Delta_{r, k} f(x)-\Delta f(x)\right| \leq \frac{1}{2}\|\Delta \Delta f\|_{\infty} r+C(M)\|f\|_{\mathcal{C}^{1}(M)} r^{-(d+3) / 2} D_{k} .
$$

Proof. As an intermediate step we define the operator $\Delta_{r}$ by

$$
\begin{aligned}
\Delta_{r} f(x) & :=\frac{1}{r}\left[\left(P_{r} f\right)(x)-f(x)\right] \\
& =\frac{1}{r}\left[\int_{M} p_{r}(x, y) f(y) d y-f(x)\right]
\end{aligned}
$$

Here $\left(P_{r}\right)_{r \geq 0}$ is the heat semigroup on $M$. A Taylor expansion at $r=0$ yields

$$
\left(P_{r} f\right)(x)=f(x)+\Delta f(x) r+\frac{1}{2} \Delta \Delta\left(P_{s} f\right)(x) r^{2}
$$

for a certain $s \in[0, r]$, hence

$$
\left|\left(P_{r} f\right)(x)-f(x)-\Delta f(x) r\right| \leq \frac{1}{2}\|\Delta \Delta f\|_{\infty} r^{2},
$$

and therefore

$$
\left|\Delta_{r} f(x)-\Delta f(x)\right| \leq \frac{1}{2}\|\Delta \Delta f\|_{\infty} r .
$$

In order to estimate the difference between $\Delta_{r}$ and $\Delta_{r, k}$ we need the following heat kernel estimate (Theorems 4 and 6 in [4]): There is a constant $\tilde{C}(M)<\infty$ such that for all $r \in(0,1]$ and all $x \in M$

$$
\left\|p_{r}(x, \cdot)\right\|_{\mathcal{C}^{1}(M)} \leq \tilde{C}(M) r^{-(d+1) / 2} .
$$

Using this estimate we obtain

$$
\begin{aligned}
\left|\Delta_{r, k} f(x)-\Delta_{r} f(x)\right| & =\frac{1}{r}\left|\frac{1}{k} \sum_{y \in S_{k}} p_{r}(x, y)[f(y)-f(x)]-\int_{M} p_{r}(x, y)[f(y)-f(x)] d y\right| \\
& \leq \frac{2}{r}\left\|p_{r}(x, \cdot)\right\|_{\mathcal{C}^{1}(M)}\|f\|_{\mathcal{C}^{1}(M)} D_{k} \\
& \leq 2 \tilde{C}(M) r^{-(d+3) / 2}\|f\|_{\mathcal{C}^{1}(M)} D_{k},
\end{aligned}
$$

and the claim follows with $C(M)=2 \tilde{C}(M)$.

We will use the following discrete version of the Euler characteristic of $M$ (motivated by the Gauss-Bonnet theorem):

$$
\chi_{k}(M):=\frac{1}{4 \pi k} \sum_{x \in S_{k}} R(x)
$$

Of course, $\left|\chi_{k}(M)-\chi(M)\right|=\frac{1}{4 \pi}\left|\frac{1}{k} \sum_{x \in S_{k}} R(x)-\int_{M} R(y) d y\right| \leq \frac{1}{4 \pi}\|R\|_{\mathcal{C}^{1}(M)} D_{k}$. 
Proposition 2. Let $\Phi(u):=\varphi(u) u$. For $f: S_{k} \rightarrow \mathbb{R}$ let

$$
K(f)(x):=f(x)+\tau\left[\Delta_{r, k}(\Phi(f))(x)-R(x)+4 \pi \chi_{k}(M) f(x)\right] .
$$

Let $t_{n}:=n \tau$,

$$
\varepsilon_{n}(x):=u\left(t_{n+1}, x\right)-K\left(u\left(t_{n}, \cdot\right)\right)(x)
$$

and

$$
\delta_{n}(x):=k \bar{X}_{n+1}(x)-K\left(k \bar{X}_{n}\right)(x) .
$$

Then there are constants $C_{1}, C_{2}, C_{3}<\infty$ (depending on $(M, \tilde{g}), R$ and $u_{0}$ ) such that for all $n \in \mathbb{N}_{0}$

$$
\left|\varepsilon_{n}(x)\right| \leq \tau\left[C_{1} \tau+C_{2} r+C_{3} r^{-5 / 2} D_{k}\right]
$$

and

Proof. Clearly,

$$
E\left[\delta_{n}(x)^{2}\right] \leq \frac{k^{2}}{4 N}
$$

$$
u\left(t_{n+1}, x\right)=u\left(t_{n}, x\right)+\int_{t_{n}}^{t_{n+1}}[\Delta(\log u)(s, x)-R(x)+4 \pi \chi(M) u(s, x)] d s,
$$

so that

$$
\begin{aligned}
\left|\varepsilon_{n}(x)\right| \leq & \int_{t_{n}}^{t_{n+1}}\left|\Delta(\log u)(s, x)-\Delta_{r, k}(\log u)\left(t_{n}, x\right)\right| d s \\
& +4 \pi \int_{t_{n}}^{t_{n+1}}\left|\chi(M) u(s, x)-\chi_{k}(M) u\left(t_{n}, x\right)\right| d s .
\end{aligned}
$$

For the first term we obtain using Lemma 2:

$$
\begin{aligned}
& \int_{t_{n}}^{t_{n+1}}\left|\Delta(\log u)(s, x)-\Delta_{r, k}(\log u)\left(t_{n}, x\right)\right| d s \\
\leq & \int_{t_{n}}^{t_{n+1}}\left|\Delta(\log u)(s, x)-\Delta(\log u)\left(t_{n}, x\right)\right| d s+\tau\left|\Delta(\log u)\left(t_{n}, x\right)-\Delta_{r, k}(\log u)\left(t_{n}, x\right)\right| \\
\leq & \tilde{C}_{1} \tau^{2}+\tau\left[C_{2} r+\tilde{C}_{3} r^{-5 / 2} D_{k}\right],
\end{aligned}
$$

where $\tilde{C}_{1}:=\frac{1}{2} \sup \left\{\frac{\partial}{\partial t} \Delta(\log u)(t, x) \mid t \in \mathbb{R}_{+}, x \in M\right\}, C_{2}:=\frac{1}{2} \sup \left\{\Delta \Delta(\log u)(t, x) \mid t \in \mathbb{R}_{+}, x \in M\right\}$ and $\tilde{C}_{3}:=C(M) \sup _{t \geq 0}\|\log u(t, \cdot)\|_{\mathcal{C} 1(M)}$. Note that these constants are finite thanks to the uniform smoothness of $u$ (see Remark 1 ). For the second term we obtain

$$
\begin{aligned}
& 4 \pi \int_{t_{n}}^{t_{n+1}}\left|\chi(M) u(s, x)-\chi_{k}(M) u\left(t_{n}, x\right)\right| d s \\
\leq & 4 \pi|\chi(M)| \int_{t_{n}}^{t_{n+1}}\left|u(s, x)-u\left(t_{n}, x\right)\right| d s+4 \pi \tau\left|\chi(M)-\chi_{k}(M)\right| u\left(t_{n}, x\right) \\
\leq & \tilde{C}_{4} \tau^{2}+\tilde{C}_{5} \tau D_{k},
\end{aligned}
$$

where $\tilde{C}_{4}:=2 \pi|\chi(M)| \sup \left\{\frac{\partial u}{\partial t}(t, x) \mid t \in \mathbb{R}_{+}, x \in M\right\}$ and $\tilde{C}_{5}:=\|R\|_{\mathcal{C}^{1}(M)}\|u\|_{L^{\infty}\left(\mathbb{R}_{+} \times M\right)}$. (These constants are finite as well by Remark 1$)$. The claim concerning $\varepsilon_{n}(x)$ follows with $C_{1}:=\tilde{C}_{1}+\tilde{C}_{4}$ and $C_{3}:=\tilde{C}_{3}+\tilde{C}_{5}$.

We now estimate $\delta_{n}(x)$ :

$$
\begin{aligned}
E\left[\delta_{n}(x)^{2}\right] & =E\left[k \bar{X}_{n+1}(x)-K\left(k \bar{X}_{n}\right)(x)\right]^{2} \\
& =E\left[k^{2} \bar{X}_{n+1}(x)^{2}\right]-2 E\left[k \bar{X}_{n+1}(x) K\left(k \bar{X}_{n}\right)(x)\right]+E\left[K\left(k \bar{X}_{n}\right)(x)^{2}\right] .
\end{aligned}
$$

The claim concerning $\delta_{n}(x)$ now follows from the following lemma. 
Lemma 3. We have

$$
E\left[k \bar{X}_{n+1}(x) K\left(k \bar{X}_{n}\right)(x)\right]=E\left[K\left(k \bar{X}_{n}\right)(x)^{2}\right]
$$

and

$$
\left|E\left[k^{2} \bar{X}_{n+1}(x)^{2}\right]-E\left[K\left(k \bar{X}_{n}\right)(x)^{2}\right]\right| \leq \frac{k^{2}}{4 N} .
$$

For the proof we need the following key lemma:

\section{Lemma 4.}

$$
\frac{k}{N} \sum_{i=1}^{N} P\left[X_{n+1}^{i}=y \mid X_{n}=\mathbf{x}\right]=K(k \overline{\mathbf{x}})(y) .
$$

Proof. We first compute $P\left[X_{n+1}^{i}=y \mid X_{n}=\mathbf{x}\right]$. In the case $y=x_{i}$ we obtain

$$
\begin{aligned}
P\left[X_{n+1}^{i}=y \mid X_{n}=\mathbf{x}\right] & =1+\tau \Delta_{r, k}\left(\varphi(k \overline{\mathbf{x}}) 1_{x_{i}}\right)(y)+\tau k^{-1} \sum_{z \in S_{k}} R(z)-\tau k^{-1} R(y) \\
& =1+\tau \Delta_{r, k}\left(\varphi(k \overline{\mathbf{x}}) 1_{x_{i}}\right)(y)+\tau 4 \pi \chi_{k}(M)-\tau k^{-1} R(y),
\end{aligned}
$$

and in the case $y \neq x_{i}$

$$
P\left[X_{n+1}^{i}=y \mid X_{n}=\mathbf{x}\right]=\tau \Delta_{r, k}\left(\varphi(k \overline{\mathbf{x}}) 1_{x_{i}}\right)(y)-\tau k^{-1} R(y),
$$

so that in both cases we obtain

$$
P\left[X_{n+1}^{i}=y \mid X_{n}=\mathbf{x}\right]=1_{\left\{y=x_{i}\right\}}+\tau\left[\Delta_{r, k}\left(\varphi(k \overline{\mathbf{x}}) 1_{x_{i}}\right)(y)+4 \pi \chi_{k}(M) 1_{\left\{y=x_{i}\right\}}-k^{-1} R(y)\right] .
$$

The claim now follows by summing over $i$.

Proof of Lemma 3. The first claim is proven as follows:

$$
\begin{aligned}
E\left[k \bar{X}_{n+1}(z) K\left(k \bar{X}_{n}\right)(z)\right] & =\sum_{\overline{\mathbf{x}}, \overline{\mathbf{y}} \in S_{k}^{N}} k \overline{\mathbf{y}}(z) K(k \overline{\mathbf{x}})(z) P\left[X_{n+1}=\mathbf{y} \text { and } X_{n}=\mathbf{x}\right] \\
& =\sum_{\overline{\mathbf{x}} \in S_{k}^{N}}\left\{\sum_{\overline{\mathbf{y}} \in S_{k}^{N}} k \overline{\mathbf{y}}(z) P\left[X_{n+1}=\mathbf{y} \mid X_{n}=\mathbf{x}\right]\right\} K(k \overline{\mathbf{x}})(z) P\left[X_{n}=\mathbf{x}\right] .
\end{aligned}
$$

Moreover,

$$
\begin{aligned}
\sum_{\overline{\mathbf{y}} \in S_{k}^{N}} k \overline{\mathbf{y}}(z) P\left[X_{n+1}=\mathbf{y} \mid X_{n}=\mathbf{x}\right] & =\frac{k}{N} \sum_{i=1}^{N} \sum_{\overline{\mathbf{y} \in S_{k}^{N}}} 1_{\left\{y_{i}=z\right\}} P\left[X_{n+1}=\mathbf{y} \mid X_{n}=\mathbf{x}\right] \\
& =\frac{k}{N} \sum_{i=1}^{N} \sum_{\substack{\overline{\mathbf{y}} \in S_{k}^{N} \\
y_{i}=z}} P\left[X_{n+1}=\mathbf{y} \mid X_{n}=\mathbf{x}\right] \\
& =\frac{k}{N} \sum_{i=1}^{N} P\left[X_{n+1}^{i}=z \mid X_{n}=\mathbf{x}\right] \\
& =K(k \overline{\mathbf{x}})(z),
\end{aligned}
$$

and the first claim follows. 
The second claim is proven as follows:

$$
\begin{aligned}
E\left[k^{2} \bar{X}_{n+1}(y)^{2}\right]= & \frac{k^{2}}{N^{2}} \sum_{i, j=1}^{N} P\left[X_{n+1}^{i}=y \text { and } X_{n+1}^{j}=y\right] \\
= & \frac{k^{2}}{N^{2}} \sum_{i, j=1}^{N} \sum_{\mathbf{x} \in S_{k}^{N}} P\left[X_{n+1}^{i}=y \text { and } X_{n+1}^{j}=y \mid X_{n}=\mathbf{x}\right] P\left[X_{n}=\mathbf{x}\right] \\
= & \frac{k^{2}}{N^{2}} \sum_{i, j=1}^{N} \sum_{\mathbf{x} \in S_{k}^{N}} P\left[X_{n+1}^{i}=y \mid X_{n}=\mathbf{x}\right] P\left[X_{n+1}^{j}=y \mid X_{n}=\mathbf{x}\right] P\left[X_{n}=\mathbf{x}\right] \\
& +\frac{k^{2}}{N^{2}} \sum_{i=1}^{N}\left[P\left[X_{n+1}^{i}=y\right]-P\left[X_{n+1}^{i}=y\right]^{2}\right] \\
= & \sum_{\mathbf{x} \in S_{k}^{N}}\left[\frac{k}{N} \sum_{i=1}^{N} P\left[X_{n+1}^{i}=y \mid X_{n}=\mathbf{x}\right]\right]^{2} P\left[X_{n}=\mathbf{x}\right] \\
& +\frac{k^{2}}{N^{2}} \sum_{i=1}^{N}\left[P\left[X_{n+1}^{i}=y\right]-P\left[X_{n+1}^{i}=y\right]^{2}\right] \\
= & \sum_{\mathbf{x} \in S_{k}^{N}}[K(k \overline{\mathbf{x}})(y)]^{2} P\left[X_{n}=\mathbf{x}\right]+\frac{k^{2}}{N^{2}} \sum_{i=1}^{N}\left[P\left[X_{n+1}^{i}=y\right]-P\left[X_{n+1}^{i}=y\right]^{2}\right] \\
= & E\left[K(k \bar{X} n)(y)^{2}\right]+\frac{k^{2}}{N^{2}} \sum_{i=1}^{N}\left[P\left[X_{n+1}^{i}=y\right]-P\left[X_{n+1}^{i}=y\right]^{2}\right] .
\end{aligned}
$$

The second claim now follows from the fact that $P(A)-P(A)^{2} \leq \frac{1}{4}$ for any event $A$.

Proposition 3. For all $n \in \mathbb{N}_{0}$,

$$
\begin{aligned}
\frac{1}{k} \sum_{x \in S_{k}}\left|u\left(t_{n}, x\right)-k \bar{X}_{n}(x)\right| \leq & \frac{1}{k} \sum_{x \in S_{k}}\left|u_{0}(x)-k \bar{X}_{0}(x)\right|+n \tau\left[C_{1} \tau+C_{2} r+C_{3} r^{-5 / 2} D_{k}\right] \\
& +\sum_{i=0}^{n-1} \frac{1}{k} \sum_{x \in S_{k}}\left|\delta_{n}(x)\right| .
\end{aligned}
$$

Proof. We write

$$
e_{n}(x):=u\left(t_{n}, x\right)-k \bar{X}_{n}(x)
$$

and

$$
a_{n}(x):=\frac{\Phi\left(u\left(t_{n}, x\right)\right)-\Phi\left(k \bar{X}_{n}(x)\right)}{u\left(t_{n}, x\right)-k \bar{X}_{n}(x)}
$$

(with the convention $\frac{0}{0}:=0$ ), so that $\Phi\left(u\left(t_{n}, x\right)\right)-\Phi\left(k \bar{X}_{n}(x)\right)=a_{n}(x) e_{n}(x)$. Note that $0 \leq$ 
$a_{n}(x) \leq 1 / u_{\min }$. Proposition 2 implies that

$$
\begin{aligned}
& e_{n+1}(x) \\
= & u\left(t_{n+1}, x\right)-k \bar{X}_{n+1}(x) \\
= & K\left(u\left(t_{n}, \cdot\right)\right)(x)-K\left(k \bar{X}_{n}\right)(x)+\varepsilon_{n}(x)-\delta_{n}(x) \\
= & e_{n}(x)+\tau \Delta_{r, k}\left(\Phi\left(u\left(t_{n}, \cdot\right)\right)-\Phi\left(k \bar{X}_{n}\right)\right)(x)+4 \pi \tau \chi_{k}(M)\left[u\left(t_{n}, x\right)-k \bar{X}_{n}(x)\right]+\varepsilon_{n}(x)-\delta_{n}(x) \\
= & \left(1+4 \pi \tau \chi_{k}(M)\right) e_{n}(x)+\frac{\tau}{r k} \sum_{y \in S_{k}} p_{r}(x, y)\left[\Phi\left(u\left(t_{n}, y\right)\right)-\Phi\left(k \bar{X}_{n}(y)\right)-\left(\Phi\left(u\left(t_{n}, x\right)\right)+\Phi\left(k \bar{X}_{n}(x)\right)\right]\right. \\
& +\varepsilon_{n}(x)-\delta_{n}(x) \\
= & \left(1+4 \pi \tau \chi_{k}(M)\right) e_{n}(x)+\frac{\tau}{r k} \sum_{y \in S_{k}} p_{r}(x, y)\left[a_{n}(y) e_{n}(y)-a_{n}(x) e_{n}(x)\right]+\varepsilon_{n}(x)-\delta_{n}(x) \\
= & {\left[1+4 \pi \tau \chi_{k}(M)-\frac{\tau}{k r} \sum_{y \in S_{k}} p_{r}(x, y) a_{n}(x)\right] e_{n}(x)+\frac{\tau}{r k} \sum_{y \in S_{k}} p_{r}(x, y) a_{n}(y) e_{n}(y)+\varepsilon_{n}(x)-\delta_{n}(x) . }
\end{aligned}
$$

Since by $(6) \tau\left[4 \pi|\chi(M)|+\frac{1}{k r} \sum_{y \in S_{k}} p_{r}(x, y) a_{n}(x)\right] \leq 1$, it follows that

$$
\begin{aligned}
\sum_{x \in S_{k}}\left|e_{n+1}(x)\right| \leq & \sum_{x \in S_{k}}\left[1-\tau 4 \pi|\chi(M)|-\tau \sum_{y \in S_{k}} p_{r}(x, y) a_{n}(x)\right]\left|e_{n}(x)\right| \\
& +\sum_{x \in S_{k}} \frac{\tau}{r k} \sum_{y \in S_{k}} p_{r}(x, y) a_{n}(y)\left|e_{n}(y)\right|+\sum_{x \in S_{k}}\left|\varepsilon_{n}(x)-\delta_{n}(x)\right| \\
= & (1-\tau 4 \pi|\chi(M)|) \sum_{x \in S_{k}}\left|e_{n}(x)\right|+\sum_{x \in S_{k}}\left|\varepsilon_{n}(x)-\delta_{n}(x)\right| .
\end{aligned}
$$

The claim follows by induction over $n$.

Proof of Theorem 1. Fix $T \geq 0, t \in[0, T]$ and $f \in B L_{1}$. Let $n:=\lfloor t / \tau\rfloor$ and $\lambda:=\lfloor t / \tau\rfloor+1-t / \tau$, so that

$$
\bar{X}_{\tau, r, k}^{N}(t, x)=\lambda \bar{X}_{n}(x)+(1-\lambda) \bar{X}_{n+1}(x) .
$$

We have

$$
\begin{aligned}
& \left|\int_{M} f(y) u(t, y) d y-\sum_{x \in S_{k}} f(x) \bar{X}_{\tau, r, k}^{N}(t, x)\right| \\
\leq & \left|\int_{M} f(y) u(t, y) d y-\frac{1}{k} \sum_{x \in S_{k}} f(x) u(t, x)\right|+\frac{1}{k} \sum_{x \in S_{k}}\left|u(t, x)-k \bar{X}_{\tau, r, k}^{N}(t, x)\right| .
\end{aligned}
$$

The first term is bounded by $\|u(t, \cdot)\|_{\mathcal{C}^{1}(M)} D_{k}$. For the second term let us note that

$$
\begin{aligned}
\left|u(t, x)-k \bar{X}_{\tau, r, k}^{N}(t, x)\right| \leq & |u(t, x)-\lambda u(n \tau, x)-(1-\lambda) u((n+1) \tau, x)| \\
& +\lambda\left|u\left(t_{n}, x\right)-k \bar{X}_{n}(x)\right|+(1-\lambda)\left|u\left(t_{n+1}, x\right)-k \bar{X}_{n+1}(x)\right|
\end{aligned}
$$

and that $|u(t, x)-\lambda u(n \tau, x)-(1-\lambda) u((n+1) \tau, x)| \leq C_{5} \tau^{2}$, where $C_{5}:=\frac{1}{8} \sup \left\{\frac{\partial^{2} u}{\partial t^{2}}(t, x) \mid t \geq\right.$ $0, x \in M\}$. Using Proposition 3 it follows that

$$
\begin{aligned}
\frac{1}{k} \sum_{x \in S_{k}}\left|u(t, x)-k \bar{X}_{\tau, r, k}^{N}(t, x)\right| \leq & C_{5} \tau^{2}+\frac{1}{k} \sum_{x \in S_{k}}\left|u_{0}(x)-k \bar{X}_{0}(x)\right| \\
& +(n+1) \tau\left[C_{1} \tau+C_{2} r+C_{3} r^{-5 / 2} D_{k}\right]+\sum_{i=0}^{n} \frac{1}{k} \sum_{x \in S_{k}}\left|\delta_{n}(x)\right| .
\end{aligned}
$$


Altogether we have

$$
\begin{aligned}
& \sup _{f \in B L_{1}} \sup _{0 \leq t \leq T}\left|\int_{M} f(y) u(t, y) d y-\sum_{x \in S_{k}} f(x) \bar{X}_{\tau, r, k}^{N}(t, x)\right| \\
\leq & \sup _{0 \leq t \leq T}\|u(t, \cdot)\|_{\mathcal{C}^{1}(M)} D_{k}+C_{5} \tau^{2}+\frac{1}{k} \sum_{x \in S_{k}}\left|u_{0}(x)-k \bar{X}_{0}(x)\right| \\
& +T\left[C_{1} \tau+C_{2} r+C_{3} r^{-5 / 2} D_{k}\right]+\sum_{i=0}^{\lceil T / \tau\rceil} \frac{1}{k} \sum_{x \in S_{k}}\left|\delta_{n}(x)\right|,
\end{aligned}
$$

and the claim follows from Proposition 2.

\section{Particle approximation for Yamabe flow}

Our method can also be applied to the non-normalized Yamabe flow

$$
\frac{\partial g}{\partial t}=-R g
$$

on a closed manifold $M$ of arbitrary dimension. The conformal class of $g$ is still conserved so that for all $t \geq 0 g_{t}$ can be written in the form $g_{t}=u(t, \cdot)^{4 /(d+2)} \tilde{g}$ with a fixed reference metric $\tilde{g}$. In dimension $d \geq 3$ the analogue of Lemma 1 is

$$
R_{g}=-\frac{4(d-1)}{d-2} \frac{1}{u} \Delta_{\tilde{g}}\left(u^{\frac{d-2}{d+2}}\right)+R_{\tilde{g}} u^{-\frac{4}{d+2}}
$$

(see e.g. [1], Corollary 1.161). This implies that

$$
\frac{\partial u}{\partial t}= \begin{cases}\frac{(d-1)(d+2)}{d-2} \Delta_{\tilde{g}}\left(u^{\frac{d-2}{d+2}}\right)-\frac{d+2}{4} R_{\tilde{g}} u^{\frac{d-2}{d+2}} & \text { if } d \geq 3 \\ \Delta_{\tilde{g}}(\log u)-R_{\tilde{g}} & \text { if } d=2 .\end{cases}
$$

In contrast to (3) the total mass of solutions of (8) is not conserved. Therefore this equation cannot be approximated by a particle system with a fixed number of particles, but one has to allow for creation or destruction of particles. In order to prevent the manifold from shrinking in a finite time, we restrict ourselves to the case $R_{\tilde{g}} \leq 0$. In this case, thanks to the maximum principle, $u$ is bounded away from 0 . We can therefore write (8) in the form

$$
\frac{\partial u}{\partial t}=\Delta_{\tilde{g}}(\varphi(u) u)-R_{\tilde{g}} \psi(u)
$$

with a smooth function $\varphi$ and a smooth, non-decreasing and concave function $\psi$.

We now consider a system consisting of a finite number of particles of mass $\frac{1}{N}$ defined on a set $S_{k}$ of $k$ points of $M$ satisfying (4). The particle dynamics takes place in discrete time steps of length $\tau>0$ and consists of two effects: particles can jump from one site to another, and independently of these jumps, new particles can be created. The jumps and the creation of particles happen in the following way: suppose that at time $n \tau$ (i.e. after $n$ time steps) there are exactly $m \geq 1$ particles at a site $x \in S_{k}$. Then each of these particles, independently of the past and of the behaviour of all other particles, behaves as follows: With probability

$$
\pi(x, y):=\frac{\tau}{k} p_{r}(x, y) \varphi(k m / N) / r
$$


it jumps to a point $y \neq x$, otherwise it stays at $x$. Moreover, independently of that and the past, new particles are created at $x$; their number is Poisson-distributed with expectation

$$
\tau \frac{N}{k}|R(x)| \psi\left(\frac{k m}{N}\right) \text {. }
$$

As in Section $2 p_{r}(x, y)$ is the heat kernel on $M$ at time $r$. In order to ensure that $\sum_{y \neq x} \pi(x, y) \leq$ 1 (so that the jump rule (9) is well-defined), and for some technical reasons we will assume that

$$
\tau \leq\left(\frac{1}{r k} \max \left(\|\varphi\|_{\infty},|\Phi|_{l i p}\right) \max _{x \in S_{k}} \sum_{y \in S_{k} \backslash\{x\}} p_{r}(x, y)\right)^{-1},
$$

where $\Phi(u):=\varphi(u) u$.

We denote the number of particles at site $x \in S_{k}$ at time $n \tau$ by $X_{n}(x)$, and we define $\bar{X}_{n}(x):=\frac{1}{N} X_{n}(x)$.

In order to study the macroscopic behaviour of the particle system we consider the empirical measure defined as

$$
\bar{X}_{\tau, r, k}^{N}(t, x):=\bar{X}_{\lfloor t / \tau\rfloor}(x)+\left(\frac{t}{\tau}-\left\lfloor\frac{t}{\tau}\right\rfloor\right)\left[\bar{X}_{\lfloor t / \tau\rfloor+1}(x)-\bar{X}_{\lfloor t / \tau\rfloor}^{N}(x)\right] .
$$

(Here we interpolate piecewise linearly in time). Then we have the following result:

Theorem 2. For all $T \geq 0$ there are constants $A_{1}, A_{2}, A_{3}, A_{4}, A_{5}<\infty$ (depending on $(M, \tilde{g})$, $u_{0}$ and $\left.T\right)$ such that for all $N, k \in \mathbb{N}$, all $r \in(0,1]$ and all $\tau>0$ satisfying (10):

$$
\begin{aligned}
& E\left[\sup _{0 \leq t \leq T} \sup _{f \in B L_{1}}\left|\int_{M} f(y) u(t, y) d y-\sum_{x \in S_{k}} f(x) \bar{X}_{\tau, r, k}^{N}(t, x)\right|\right] \\
\leq & A_{1} E\left[\frac{1}{k} \sum_{x \in S_{k}}\left|u_{0}(x)-k \bar{X}_{0}(x)\right|\right]+A_{2} \tau+A_{3} r+A_{4} r^{-(d+3) / 2} D_{k}+A_{5} \frac{k}{\tau \sqrt{N}} .
\end{aligned}
$$

This means that the empirical measure of the particle system converges locally uniformly in time to the measure with density $u$ as $N$ and $k$ tend to $\infty$ and $\tau$ and $r$ tend to 0 , provided that the initial configuration is chosen appropriately and the constraints $r^{-(d+3) / 2} D_{k} \rightarrow 0, \frac{k}{\tau \sqrt{N}} \rightarrow 0$ and (10) are respected.

The proof of Theorem 2 is quite similar to the one of Theorem 1. We start with an analogue of Lemma 2:

Proposition 4. Let $\Phi(u):=\varphi(u) u$. For $f: S_{k} \rightarrow \mathbb{R}$ let

$$
K(f)(x):=f(x)+\tau\left[\Delta_{r, k}(\Phi(f))(x)-R(x) \psi(f(x))\right] .
$$

Let $t_{n}:=n \tau$,

$$
\varepsilon_{n}(x):=u\left(t_{n+1}, x\right)-K\left(u\left(t_{n}, \cdot\right)\right)(x)
$$

and

$$
\delta_{n}(x):=k \bar{X}_{n+1}(x)-K\left(k \bar{X}_{n}\right)(x) .
$$

Then for all $T \geq 0$ there are constants $C_{1}, C_{2}, C_{3}, C_{4}<\infty$ (depending on $(M, \tilde{g}), R, u_{0}$ and $T$ ) such that for all $n \leq T / \tau$ :

$$
\left|\varepsilon_{n}(x)\right| \leq \tau\left[C_{1} \tau+C_{2} r+C_{3} r^{-(d+3) / 2} D_{k}\right]
$$

and

$$
E\left[\delta_{n}(x)^{2}\right] \leq C_{4} \frac{k^{2}}{N}
$$


Proof. The claim concerning $\varepsilon_{n}(x)$ is proven as the corresponding claim in Proposition 2 .

In order to estimate $\delta_{n}(x)$ let us observe that the particles which are at $x$ after $n+1$ time steps belong to three different classes:

1. those which were at another site $z \neq x$ at time $n \tau$,

2. those which were at $x$ at time $n \tau$, and

3. those which were created in the last time step.

We denote their respective numbers by $X_{n+1}^{1, z}(x), X_{n+1}^{2}(x)$ and $X_{n+1}^{3}(x)$. By definition of the particle system, conditioned on $X_{n}=X$, these quantities are independent and distributed as follows:

$$
\begin{aligned}
X_{n+1}^{1, z}(x) & \sim B\left(X(z), \tau \frac{p_{r}(z, x)}{k r} \varphi(k \bar{X}(z))\right), \\
X_{n+1}^{2}(x) & \sim B\left(X(x), 1-\sum_{z \neq x} \tau \frac{p_{r}(x, z)}{k r} \varphi(k \bar{X}(x))\right), \\
X_{n+1}^{3}(x) & \sim \pi\left(-\tau \frac{N}{k} R(x) \psi(k \bar{X}(x))\right) .
\end{aligned}
$$

Here $B(n, p)$ and $\pi(\lambda)$ denote the binomial and the Poisson distribution, respectively.

It follows that

$$
\begin{aligned}
& E\left[k \bar{X}_{n+1}(x) \mid X_{n}=X\right] \\
= & \frac{k}{N}\left[\sum_{z \neq x} E\left[X_{n+1}^{1, z}(x) \mid X_{n}=X\right]+E\left[X_{n+1}^{2}(x) \mid X_{n}=X\right]+E\left[X_{n+1}^{3}(x) \mid X_{n}=X\right]\right] \\
= & \frac{k}{N}\left[\sum_{z \neq x} X(z) \tau \frac{p_{r}(z, x)}{k r} \varphi(k \bar{X}(z))+X(x)\left[1-\sum_{z \neq x} \tau \frac{p_{r}(x, z)}{k r} \varphi(k \bar{X}(x))\right]-\tau \frac{N}{k} R(x) \psi(k \bar{X}(x))\right] \\
= & \frac{k}{N}\left[X(x)+\tau \sum_{z \neq x} \frac{p_{r}(x, z)}{k r}[X(z) \varphi(k \bar{X}(z))-X(x) \varphi(k \bar{X}(x))]-\tau \frac{N}{k} R(x) \psi(k \bar{X}(x))\right] \\
= & k \bar{X}(x)+\tau \sum_{z \neq x} \frac{p_{r}(x, z)}{k r}[k \bar{X}(z) \varphi(k \bar{X}(z))-k \bar{X}(x) \varphi(k \bar{X}(x))]-\tau R(x) \psi(k \bar{X}(x)) \\
= & k \bar{X}(x)+\tau\left[\Delta_{r, k}(\Phi(k \bar{X}))(x)-R(x) \psi(k \bar{X}(x))\right] \\
= & K(k \bar{X})(x) .
\end{aligned}
$$


Moreover, using Bienaymé's equality and the fact that $p(1-p) \leq 1 / 4$ for every $p \in[0,1]$,

$$
\begin{aligned}
& \operatorname{Var}\left(k \bar{X}_{n+1}(x) \mid X_{n}=X\right) \\
= & \frac{k^{2}}{N^{2}} \operatorname{Var}\left(X_{n+1}(x) \mid X_{n}=X\right) \\
= & \frac{k^{2}}{N^{2}}\left[\sum_{z \neq x} \operatorname{Var}\left[X_{n+1}^{1, z}(x) \mid X_{n}=X\right]+\operatorname{Var}\left[X_{n+1}^{2}(x) \mid X_{n}=X\right]+\operatorname{Var}\left[X_{n+1}^{3}(x) \mid X_{n}=X\right]\right] \\
\leq & \frac{k^{2}}{N^{2}}\left[\sum_{z \neq x} X(z) \frac{1}{4}+X(x) \frac{1}{4}-\tau \frac{N}{k} R(x) \psi(k \bar{X}(x))\right] \\
= & \frac{k^{2}}{4 N} \sum_{z \in S_{k}} \bar{X}(z)-\tau \frac{k}{N} R(x) \psi(k \bar{X}(x)) .
\end{aligned}
$$

It follows that

$$
\begin{aligned}
& E\left[\delta_{n}(x)^{2}\right] \\
= & E\left[\left(k \bar{X}_{n+1}(x)-K\left(k \bar{X}_{n}(x)\right)\right)^{2}\right] \\
= & \sum_{X \in \mathbb{N}_{0}^{S_{k}}} E\left[\left(k \bar{X}_{n+1}(x)-K\left(k \bar{X}_{n}(x)\right)\right)^{2} \mid X_{n}=X\right] P\left[X_{n}=X\right] \\
= & \sum_{X \in \mathbb{N}_{0}^{S_{k}}} \operatorname{Var}\left(k \bar{X}_{n+1}(x) \mid X_{n}=X\right) P\left[X_{n}=X\right] \\
\leq & \sum_{X \in \mathbb{N}_{0}^{S_{k}}}\left[\frac{k^{2}}{4 N} \sum_{z \in S_{k}} \bar{X}(z)-\tau \frac{k}{N} R(x) \psi(k \bar{X}(x))\right] P\left[X_{n}=X\right] \\
= & E\left[\frac{k^{2}}{4 N} \sum_{z \in S_{k}} \bar{X}_{n}(z)-\tau \frac{k}{N} R(x) \psi\left(k \bar{X}_{n}(x)\right)\right] .
\end{aligned}
$$

Since by the following lemma $E\left[\sum_{x \in S_{k}} \bar{X}_{n}(x)\right] \leq C(T)$ the claim follows.

Lemma 5. Let

$$
\bar{\psi}(u):=\int_{0}^{u} \frac{1}{\psi(x)} d x .
$$

Then

$$
E\left[\sum_{x \in S_{k}} \bar{X}_{n}(x)\right] \leq \bar{\psi}^{-1}\left(\bar{\psi}\left(E\left[\sum_{x \in S_{k}} \bar{X}_{0}(x)\right]\right)+\|R\|_{\infty} n \tau\right) .
$$


Proof.

$$
\begin{aligned}
E\left[\sum_{x \in S_{k}} \bar{X}_{n+1}(x)\right] & =\frac{1}{k} \sum_{x \in S_{k}} E\left[k \bar{X}_{n+1}(x)\right] \\
& =\frac{1}{k} \sum_{x \in S_{k}} E\left[K\left(k \bar{X}_{n}\right)(x)\right] \\
& =\frac{1}{k} \sum_{x \in S_{k}} E\left[k \bar{X}_{n}(x)+\tau \Delta_{r, k}\left(\Phi\left(k \bar{X}_{n}\right)\right)(x)-\tau R(x) \psi\left(k \bar{X}_{n}(x)\right)\right] \\
& =E\left[\sum_{x \in S_{k}} \bar{X}_{n}(x)\right]+\frac{\tau}{k} \sum_{x \in S_{k}}|R(x)| E\left[\psi\left(k \bar{X}_{n}(x)\right)\right]
\end{aligned}
$$

Since $\psi$ is concave it follows that

$$
\begin{aligned}
E\left[\sum_{x \in S_{k}} \bar{X}_{n+1}(x)\right]-E\left[\sum_{x \in S_{k}} \bar{X}_{n}(x)\right] & \leq\|R\|_{\infty} \tau \frac{1}{k} \sum_{x \in S_{k}} E\left[\psi\left(k \bar{X}_{n}(x)\right)\right] \\
& \leq\|R\|_{\infty} \tau \psi\left(\frac{1}{k} \sum_{x \in S_{k}} E\left[k \bar{X}_{n}(x)\right]\right) \\
& =\|R\|_{\infty} \tau \psi\left(\sum_{x \in S_{k}} E\left[\bar{X}_{n}(x)\right]\right) .
\end{aligned}
$$

Moreover, since $\bar{\psi}^{\prime}=\frac{1}{\psi}$ and because $\psi$ is nondecreasing,

$$
\begin{aligned}
& \bar{\psi}\left(E\left[\sum_{x \in S_{k}} \bar{X}_{n}(x)\right]\right)-\bar{\psi}\left(E\left[\sum_{x \in S_{k}} \bar{X}_{n-1}(x)\right]\right) \\
\leq & \frac{1}{\psi\left(E\left[\sum_{x \in S_{k}} \bar{X}_{n-1}(x)\right]\right)}\left[E\left[\sum_{x \in S_{k}} \bar{X}_{n}(x)\right]-E\left[\sum_{x \in S_{k}} \bar{X}_{n-1}(x)\right]\right] \\
\leq & \frac{1}{\psi\left(E\left[\sum_{x \in S_{k}} \bar{X}_{n-1}(x)\right]\right)}\|R\|_{\infty} \tau \psi\left(\sum_{x \in S_{k}} E\left[\bar{X}_{n-1}(x)\right]\right) \\
= & \|R\|_{\infty} \tau .
\end{aligned}
$$

The claim now follows by induction.

Proposition 5. For all $n \leq T / \tau$ :

$$
\begin{aligned}
\frac{1}{k} \sum_{x \in S_{k}}\left|u\left(t_{n}, x\right)-k \bar{X}_{n}(x)\right| \leq & \left(1+\tau\|R\|_{\infty}|\psi|_{l i p}\right)^{n} \frac{1}{k} \sum_{x \in S_{k}}\left|u_{0}(x)-k \bar{X}_{0}(x)\right| \\
& +\sum_{i=0}^{n-1}\left(1+\tau\|R\|_{\infty}|\psi|_{l i p}\right)^{n-1-i} \frac{1}{k} \sum_{x \in S_{k}}\left|\varepsilon_{i}(x)-\delta_{i}(x)\right| .
\end{aligned}
$$

Proof. As in the proof of Proposition 3 we write

$$
e_{n}(x):=u\left(t_{n}, x\right)-k \bar{X}_{n}(x),
$$




$$
a_{n}(x):=\frac{\Phi\left(u\left(t_{n}, x\right)\right)-\Phi\left(k \bar{X}_{n}(x)\right)}{u\left(t_{n}, x\right)-k \bar{X}_{n}(x)}
$$

and

$$
b_{n}(x):=\frac{\psi\left(u\left(t_{n}, x\right)\right)-\psi\left(k \bar{X}_{n}(x)\right)}{u\left(t_{n}, x\right)-k \bar{X}_{n}(x)}
$$

(with the convention $\frac{0}{0}:=0$ ) and obtain

$$
\begin{aligned}
e_{n+1}(x)= & {\left[1-\tau R(x) b_{n}(x)-\frac{\tau}{k r} \sum_{y \in S_{k}} p_{r}(x, y) a_{n}(x)\right] e_{n}(x) } \\
& +\frac{\tau}{r k} \sum_{y \in S_{k}} p_{r}(x, y) a_{n}(y) e_{n}(y)+\varepsilon_{n}(x)-\delta_{n}(x) .
\end{aligned}
$$

Since by $(10) \frac{\tau}{k r} \sum_{y \in S_{k}} p_{r}(x, y) a_{n}(x) \leq 1$, it follows that

$$
\begin{aligned}
\sum_{x \in S_{k}}\left|e_{n+1}(x)\right| \leq & \sum_{x \in S_{k}}\left[1-\tau R(x) b_{n}(x)-\frac{\tau}{r k} \sum_{y \in S_{k}} p_{r}(x, y) a_{n}(x)\right]\left|e_{n}(x)\right| \\
& +\sum_{x \in S_{k}} \frac{\tau}{r k} \sum_{y \in S_{k}} p_{r}(x, y) a_{n}(y)\left|e_{n}(y)\right|+\sum_{x \in S_{k}}\left|\varepsilon_{n}(x)-\delta_{n}(x)\right| \\
= & \sum_{x \in S_{k}}\left[1-\tau R(x) b_{n}(x)\right]\left|e_{n}(x)\right|+\sum_{x \in S_{k}}\left|\varepsilon_{n}(x)-\delta_{n}(x)\right| \\
\leq & {\left[1+\tau\|R\|_{\infty}|\psi|_{l i p}\right] \sum_{x \in S_{k}}\left|e_{n}(x)\right|+\sum_{x \in S_{k}}\left|\varepsilon_{n}(x)-\delta_{n}(x)\right| }
\end{aligned}
$$

The claim follows by induction over $n$.

The proof of Theorem 2 can now be concluded in a similar way as the proof of Theorem 1 .

\section{References}

[1] Besse, A., Einstein Manifolds, Springer-Verlag, Berlin, Heidelberg, New York, 1987.

[2] Brendle, S., Convergence of the Yamabe flow for arbitrary initial energy, J. Differ. Geom. 69 (2005), 217-278.

[3] Brendle, S., Convergence of the Yamabe flow in dimension 6 and higher, Invent. Math. 170 (2007), 541-576.

[4] Cheng, S. Y., Li, P. and Yau, S.-T., On the upper estimate of the heat kernel of a complete Riemannian manifold, Amer. J. Math. 103 (1981), 1021-1063.

[5] Chow, B., The Ricci flow on the 2-sphere, J. Differ. Geom. 33 (1991), 325-334.

[6] Chow, B. and Knopf, D., The Ricci Flow: An Introduction, American Mathematical Society, Providence, RI, 2004.

[7] Chow, B. and Luo, F., Combinatorial Ricci flows on surfaces, J. Differ. Geom. 63 (2003), 97-129.

[8] Dudley, R. M., Real Analysis and Probability, Cambridge University Press, 2002. 
[9] Ekhaus, M. and Seppäläinen, T., Stochastic dynamics macroscopically governed by the porous medium equation for isothermal flow, Ann. Acad. Sci. Fenn., Math. 21 (1996), 309-352.

[10] Feng, S., Iscoe, I. and Seppäläinen, T., A microscopic mechanism for the porous medium equation, Stochastic Processes Appl. 66 (1997), 147-182.

[11] Figalli, A. and Philipowski, R., Convergence to the porous medium equation and propagation of chaos, Alea 4 (2008), 185-203.

[12] Hamilton, R. S., The Ricci flow on surfaces, in: Isenberg, J. A. (Ed.), Mathematics and General Relativity (American Mathematical Society, Providence, RI, 1988), 237-262.

[13] Inoue, M., Derivation of a porous medium equation from many Markovian particles and the propagation of chaos, Hiroshima Math. J. 21 (1991), 85-110.

[14] Jourdain, B., Probabilistic approximation for a porous medium equation, Stochastic Processes Appl. 89 (2000), 81-99.

[15] Kipnis, C. and Landim, C., Scaling Limits of Interacting Particle Systems, Springer-Verlag, Berlin, Heidelberg, New York, 1999.

[16] Luo, F., Combinatorial Yamabe flows on surfaces, Comm. Contemp. Math. 6 (2004), 765780 .

[17] Morgan, J. and Tian, G., Ricci Flow and the Poincaré Conjecture, American Mathematical Society, Providence, RI, 2007.

[18] Oelschläger, K., Large systems of interacting particles and the porous medium equation, $J$. Differ. Equations 88 (1990), 294-346.

[19] Perelman, G., The entropy formula for the Ricci flow and its geometric applications, arXiv:math.DG/0211159 (2002).

[20] Perelman, G., Ricci flow with surgery on three-manifolds, arXiv:math.DG/0303109 (2003).

[21] Perelman, G., Finite extinction time for the solutions to the Ricci flow on certain threemanifolds, arXiv:math.DG/0307245 (2003).

[22] Philipowski, R., Interacting diffusions approximating the porous medium equation and propagation of chaos, Stochastic Processes Appl. 117 (2007), 526-538.

[23] Schwetlick, H. and Struwe, M., Convergence of the Yamabe flow for "large" energies, J. Reine Angew. Math. 562 (2003), 59-100.

[24] Spohn, H., Large Scale Dynamics of Interacting Particles, Springer-Verlag, Berlin, Heidelberg, New York, 1991.

[25] Vázquez, J. L., The Porous Medium Equation: Mathematical Theory, Clarendon Press, Oxford, 2007.

[26] Ye, R., Global existence and convergence of Yamabe flow, J. Differ. Geom. 39 (1994), $35-50$. 